\title{
LHCb semileptonic highlights from 2010 and prospects for 2011
}

\author{
Robert W. Lambert ${ }^{* t}$ \\ CERN, Geneva, Switzerland \\ E-mail: Rob. Lambert@cern.ch
}

The LHCb experiment collected $\sim 38 \mathrm{pb}^{-1}$ of $p p$-collisions at a centre-of-mass energy of $7 \mathrm{TeV}$ in 2010. With such a data sample we are able to measure several properties of semileptonic $b$-decays, such as the exclusive branching ratios of $B_{s / d}^{0} \rightarrow D^{(*)(*)} \mu v$ channels necessary to determine $V_{c b}$, and later measure $V_{u b}$. I present a summary of the work so far on semileptonic $b$-decays, including the first observation of $B_{s} \rightarrow D_{s 2}^{*+} X \mu v$, and also the prospects for the measurement of the flavourspecific asymmetry, $a_{f s}$ in 2011.

The 13th International Conference on B-Physics at Hadron Machines - Beauty2011, April 04-08, 2011

Amsterdam, The Netherlands

\footnotetext{
* Speaker.

$\dagger$ On behalf of the LHCb collaboration.
} 


\section{Introduction}

The LHCb $B$-hadron samples will rapidly overtake the samples collected to date by the $B$ factories and the Tevatron. Semileptonic $B$-decays are a very abundant class of $B$-decays. Competitive measurements and new results in the $B_{s}^{0}$-system, have arisen even from the initial LHC run period (2010), where we collected $\sim 38 \mathrm{pb}^{-1}$ of integrated luminosity at a centre-of-mass energy of $7 \mathrm{TeV}$. By the end of 2011 we expect to have collected $1 \mathrm{fb}^{-1}$, and these will be sufficient data for many detailed analyses. In this brief paper we cover a few of the interesting topics for 2010 and 2011 analyses of these data.

\subsection{The LHCb detector}

The $\mathrm{LHCb}$ detector is a single forward-arm spectrometer described in detail elsewhere [1]. The key components of LHCb which are significant for semileptonic studies, and differ considerably from previous experiments include the LHCb vertex locator (VELO) and the PID subsystems. The VELO is a silicon strip device approaching to within $8 \mathrm{~mm}$ of the beam line, that can provide excellent proper-time resolution of $\sim 50 \mathrm{fs}$ for fully reconstructed final states. The dedicated PID subsystems, such as the two RICH detectors, Muon stations and Calorimeters, enable LHCb to efficiently separate final state particles, notably including $K / \pi$ mesons, enabling many formerly impossible exclusive studies.

\subsection{Semileptonic studies at LHCb}

We are reconstructing and analysing many different semileptonic $B$-decay modes, which can be well-separated at LHCb. The main backgrounds are from open charm (prompt charm) production at the primary vertex (interaction point), and other light meson production. Here we pick two of the many examples of decays reconstructed in LHCb in Fig. 1 as a demonstration of detector performance, namely the decay $\Lambda_{b}^{+} \rightarrow \Lambda_{c}^{+} \mu \nu X$ and inclusive $X_{b} \rightarrow D^{+} \mu \nu X$.
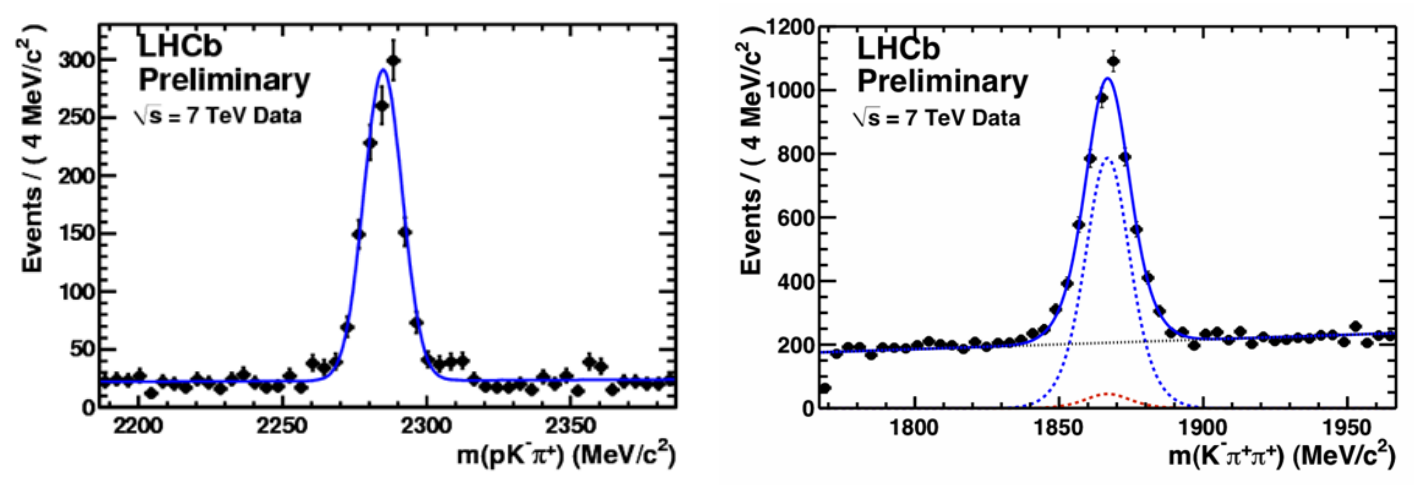

Figure 1: Two of the many examples of semileptonic decays reconstructed in LHCb, namely $\Lambda_{b}$ (left), where we plot the mass distribution of the $(p K \pi)$ from the subsequent $\Lambda_{c}$-decay, and $X_{b} \rightarrow D^{+} \mu v X$ (right), where the $K \pi \pi$ mass distribution is plotted. These plots were created from an integrated luminosity of $\sim 0.5 \mathrm{pb}^{-1}$. One may see that the combinatorial background is effectively suppressed. In black, the data; in solid blue, the total fit; in dashed blue, the detached-vertex-signal; in dashed red, the prompt peaking background which is separated using the impact parameter (IP) distibution; in dashed black, the combinatorial background. 
The physics programme of LHCb's semileptonic studies is very broad, and can be classified into two main areas, time-dependent and time-integrated studies. Time-dependent measurements provide sensitivity to $\Gamma, \Delta m, \Delta \Gamma$ and $a_{f s}$. Time-integrated measurements provide sensitivity to cross-sections, branching-ratios, form-factors, $a_{f s}$, and CKM-parameters such as $V_{u b}$ and $V_{c b}$. In this paper we concentrate on the time-independent studies, and briefly touch upon time-dependent determination of $a_{f s}$.

The first LHCb $b$-physics paper, Ref. [2], is the determination of the $b \bar{b}$-cross-section from decays of the form $X_{b} \rightarrow D^{0} \mu v X$. In that paper we use the impact parameter (IP) distribution to effectively separate signal from background. Open charm and other combinatorics tend to be produced at the pp-interaction point (primary vertex), with small IP. We can fit to the IP distribution of the reconstructed meson with respect to the primary vertex to separate out the detached signal from the prompt background.

\subsection{Towards $V_{u b}$ and $V_{c b}$ measurements}

One key physics goal we can identify is a precise measurement of $V_{u b}$ and $V_{c b}$ in the $B_{s}^{0}$-system. $V_{u b}$ is an interesting parameter to measure for several reasons [3]. $V_{u b}$ constrains the length of the side of the unitarity triangle (UT) opposite the angle $\beta$, and so is an independent constraint of the corner of the UT. It is measured in tree-level $b \rightarrow u$ decays; however, even the most recent measurements do not provide a significant constraint on the UT fit [4]. $V_{u b}$ is also very useful in separating out many different theoretical models and form factor descriptions. The BABAR collaboration measure $V_{u b}$ with $B_{d}^{0}$, in bins of $q^{2}$ [5], and use this to discriminate different form factor models. In the $B_{s}^{0}$ system very little is currently known.

Decays involving $V_{c b}$ form the main background to a measurement of $V_{u b}$, this is one reason we must first understand decays involving $V_{c b}$. This class of decays are also interesting experimentally since very little is known of either of the two inputs required to understand the $B_{s}^{0}$ system, namely: the exclusive branching ratios of many different final states, and the hadronic form factors. The majority of the relevant form factors have yet to be calculated on the lattice ${ }^{1}$, and there are only weak experimental constraints on the exclusive $D_{s}$ and $D_{s}^{*(*)}$ branching ratios [6]. Particularly the branching ratios and form factors for the excited $D_{s}^{*(*)}$ decays are very important, as they are expected to contribute the majority of the total branching ratio [7]. The experimental determination of branching ratios for the $D^{* *}$ decays are a crucial first step in the attempt on $V_{c b}$ and $V_{u b}$, and we have measured two such ratios at LHCb.

\section{Measurement of $D_{s 1}^{+}$and first observation of $D_{s 2}^{*+}$}

Due to the rapidly changing collider and experimental conditions we divide the data sample into two parts. For the first $3 \mathrm{pb}^{-1}$ we take events which have passed the single muon trigger. These events nominally have a low number of primary vertices per event. For the second sample of $20 \mathrm{pb}^{-1}$ we take all triggered events, since the single muon trigger was heavily prescaled by a factor 50 to cope with the quickly increasing LHC luminosity. In the larger sample, due to this prescaling, it would be much more difficult to normalize to the total semileptonic rate, and so the analysis takes the following steps:

\footnotetext{
1 although we understand, from this conference, work has begun in that area
} 


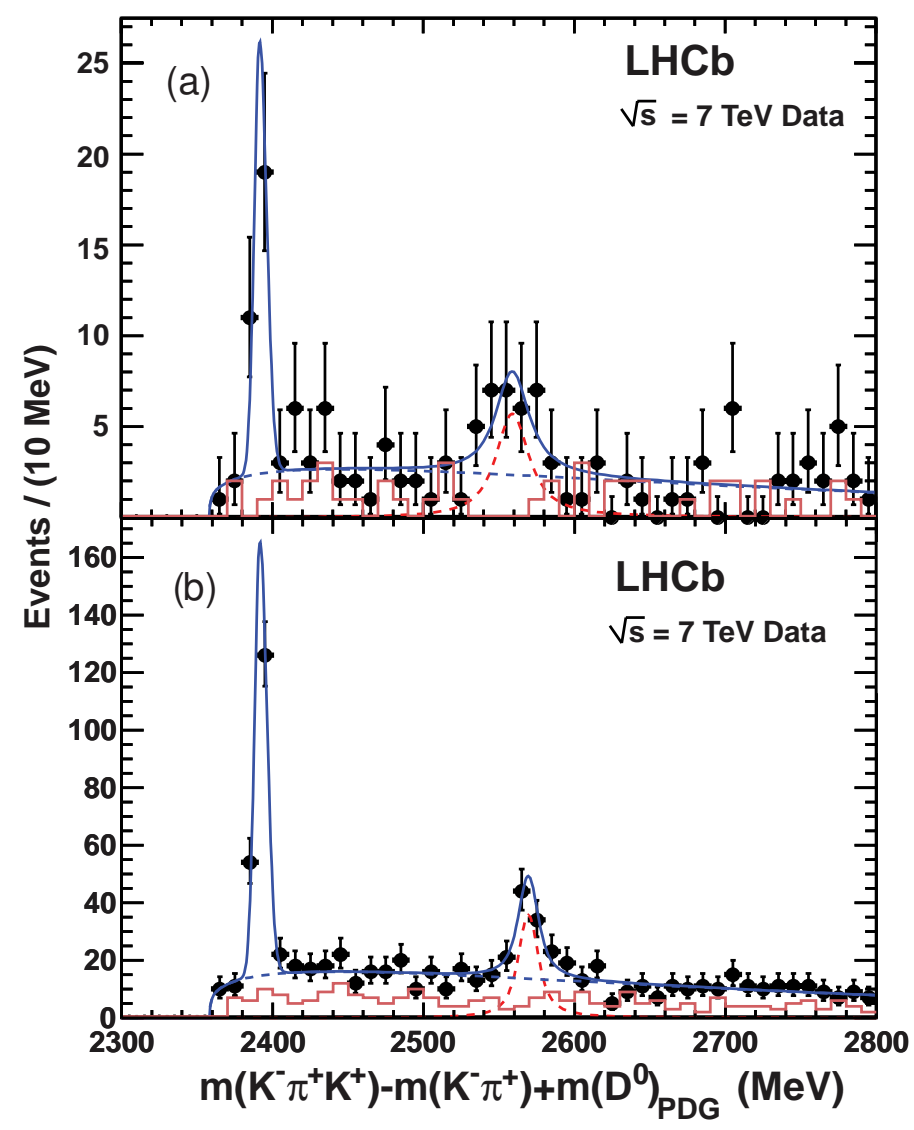

Figure 2: The mass difference $m\left(K^{-} \pi^{+} K^{+}\right)-m\left(K^{-} \pi^{+}\right)$added to the known $D^{0}$ mass for events with $K^{-} \pi^{+}$ invariant masses within $\pm 20 \mathrm{MeV}$ of the $D^{0}$ mass (black points) in semileptonic decays from Ref. [8]. The histogram shows wrong-sign events with an additional $K^{-}$instead of a $K^{+}$. (a) For the $3 \mathrm{pb}^{-1}$ data sample and (b) for the $20 \mathrm{pb}^{-1}$ sample. The curves are described in Ref. [8]. The first peak is consistent with the known $B_{s}^{0} \rightarrow D_{s 1}^{+} X \mu \nu$ resonance, the second peak is the first observation of the $B_{s}^{0} \rightarrow D_{s 2}^{*+} X \mu \nu$ decay, consistent with the known $D_{s 2}^{*+}$-mass.

- Observe resonances in $D^{0} K \mu$ in both samples

- Measure significances, widths and masses in the larger $20 \mathrm{pb}^{-1}$ sample

- Measure the relative branching ratios of resonances in the larger $20 \mathrm{pb}^{-1}$ sample

- Normalize to the total semileptonic rate in the smaller $3 \mathrm{pb}^{-1}$, which can be determined accurately due to the inclusive triggering

A sample of $B_{s}^{0} \rightarrow D_{s}^{* *} \mu X$ decays is selected by searching for a charmed meson which forms a common vertex with a kaon and muon. Cuts were optimised for the signal to background ratio in simulation. We require well-fitted tracks, a well-isolated well-identified muon, well-identified hadrons and well-reconstructed vertices which point back to an interaction vertex (primary vertex). The selection is fully described in Ref. [8]. The yield is shown as a function of mass in Fig. 2. 
A $D_{s 2}^{*+}$-resonance is observed at $>8 \sigma$ in the larger dataset, which is the first observation of this decay channel. Combining the samples we measure the ratio of branching fractions to be

$$
\frac{B\left(B_{s}^{0} \rightarrow D_{s 2}^{*+} X \mu v\right)}{B\left(B_{s}^{0} \rightarrow X \mu v\right)}=(3.3 \pm 1.0 \pm 0.4) \%
$$

widths and masses are also measured, for further details see Ref. [8].

Amongst other uses, this measurement is a crucial input to our Monte Carlo simulation, to constrain the ratio of $D_{s}^{* *}$ states to $D_{s}$ and $D_{s}^{*}$, necessary for $V_{c b}$, and also for measuring timedependent parameters in semileptonic $B_{s}^{0}$-decays such as flavour-specific asymmetry, $a_{f s}$.

\section{Prospects for $a_{f s}$}

The $\mathrm{D} \varnothing$ collaboration produced an exciting and surprizing result in the measurement of flavourspecific asymmetry in the semileptonic decays of $b$-quarks [9], which is 3.2 standard deviations from the standard model prediction. This result has sparked great interest and has been extensively discussed elsewhere in this conference.

Fig. 3 is reproduced from Ref. [9] and slightly modified to also include the expected $\mathrm{LHCb}$ sensitivity taken from simulation (Monte Carlo or MC), applying the real-data yields and estimates of systematic uncertainties. In the environment of the LHC, such a measurement is made more challenging by the expected production asymmetry [10], however, using a novel time-dependent technique LHCb can make an accurate measurement of $\Delta A_{f s}=\left(a_{f s}^{s}-a_{f s}^{d}\right) / 2$, with a statistical sensitivity (as predicted from the MC) of $\approx 2 \times 10^{-3}$ in $1 \mathrm{fb}^{-1}$ [11]. This measurement is complementary to the $\mathrm{D} \emptyset$ measurement, and almost orthogonal in the $\left(a_{f_{s}}^{s}: a_{f_{s}}^{d}\right)$-plane.
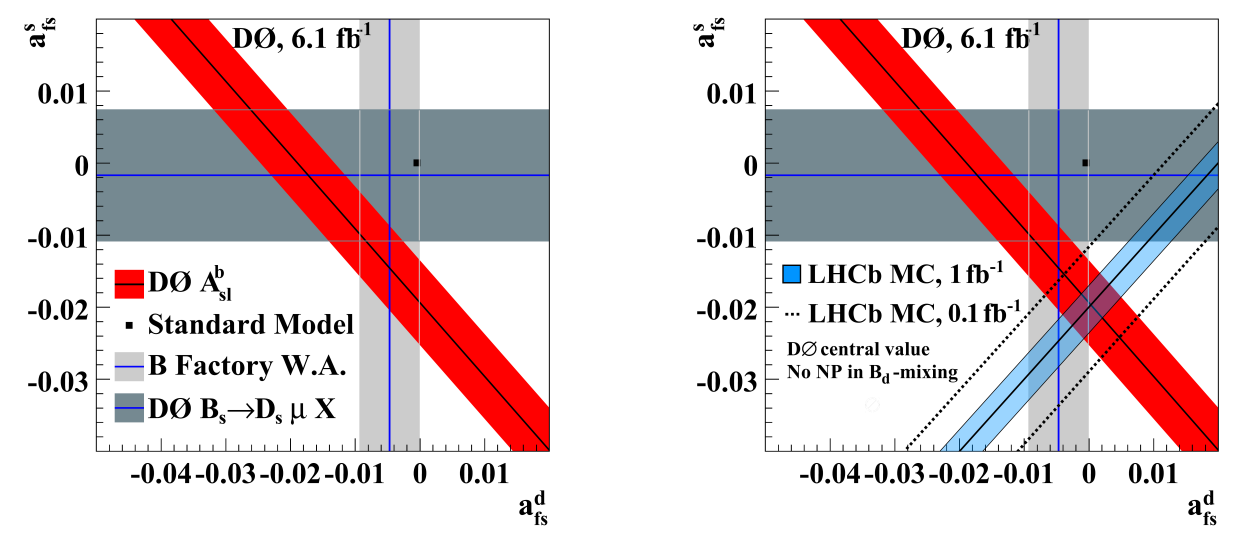

Figure 3: Measurements and prospects for new physics in flavour-specific asymmetry. The asymmetry in neutral $B^{0}$ mixing, $a_{f s}^{d}$ is plotted against the asymmetry in neutral $B_{s}^{0}$-mixing, $a_{f s}^{s}$. The left figure is reproduced from Ref. [9] (slightly modified), the recent $\mathrm{D} \emptyset$ measurement in red is the first measurement inconsistent with the standard model point of $\sim(0,0)$. The right figure also has superimposed the LHCb expected result from simulation (Monte Carlo), should the $\mathrm{D} \emptyset$ central value hold and should there be no new physics in $a_{f s}^{d}$. There we scale the Monte Carlo (MC) prediction [11] to the yields in real data and add also the expected systematic uncertainties. Notably, $1 \mathrm{fb}^{-1}$ is the expected 2011 dataset. 


\section{Conclusion}

LHC and LHCb are performing excellently, and we have collected $\sim 38 \mathrm{pb}^{-1}$ of $p p$-collision data at $\sqrt{s}=7 \mathrm{TeV}$ in the 2010 run period. With these data we are beginning to understand the detector and constrain models of form factors and exclusive branching ratios of $B_{s}^{0} \rightarrow D^{(*)(*)} \mu \nu$ states, necessary for a measurement of $V_{c b}$ and then $V_{u b}$. In 2011 we expect to collect $1 \mathrm{fb}^{-1}$, which will allow us to make clean measurements within many time-integrated and time-dependent topics including $V_{c / u b}$ and $\Delta A_{f s}=\left(a_{f s}^{s}-a_{f s}^{d}\right) / 2$.

\section{Acknowledgements}

Thanks to the LHC and the rest of the LHCb collaboration for providing the data we are analysing at the moment, and special thanks to my close colleagues: Marina Artuso, Phillip Urquijo, Kim Vervink, Liming Zhang for direct contributions to this talk.

\section{References}

[1] A. Augusto Alves et al., LHCb collaboration, The LHCb detector at LHC, J. of Instrumentation 3 (2008) pp. S08005.

[2] R. Aaij et al., the LHCb collaboration, "Measurement of sigma(pp -> b anti-b X) at $\sqrt{s}=7 \mathrm{TeV}$ in the forward region,” Phys. Lett. B 694 (2010) pp. 209.

[3] R. W. Lambert, on behalf of the LHCb Collaboration, "Semileptonic $B$-decays at LHCb, and the Prospects for measuring $V_{c b}$ and $V_{u b}$," in Proceedings of the 10th International Conference on Heavy Quarks and Leptons, HQL 2010, INFN, Frascati, Italy, 11-15 October 2010, to be published in Proc. of Sci. (HQL 2010), CERN-LHCb-PROC-2011-009 (2011).

[4] J. Charles et al., the CKMfitter Group, "CP violation and the CKM matrix: assessing the impact of the asymmetric $B$ factories," Eur. Phys. J. C41, 1-131 (2005), hep-ph 0406184, updated results and plots available at: http://ckmfitter.in2p3.fr.

[5] B. Aubert et al., the BABAR collaboration, "Study of $B \rightarrow \pi l v$ and $B \rightarrow \rho l v$ decays and determination of $\left|V_{u b}\right|$," to be published in Phys. Rev. D, hep-ex arxiv:1005.3288.

[6] V.M. Abazov et al., the DØ Collaboration, "Measurement of the semileptonic branching ratio of $B_{s}^{0}$ to an orbitally excited $D_{s}^{* *}$ state $\operatorname{Br}\left(B_{s}^{0} \rightarrow D_{s 1}^{-}(2536) \mu^{+} v X\right)$," Phys. Rev. Lett.102 (2009) pp. 051801.

[7] J.-M. Zhang and G.-L. Wang, " $B_{s}$ semileptonic decays to $D_{s}$ and $D_{s}^{*}$ in Bethe-Salpeter method," Chin. Phys. Lett. 27 (2010) pp. 051301, hep-ph arxiv:1003.5576.

[8] R. Aaij et al., the LHCb collaboration, "First observation of $B_{s} \rightarrow D_{s 2}^{*+} X \mu \nu$ decays," Phys. Lett. B 698 (2011) pp. 14-20.

[9] V. M. Abazov et al., the $\mathrm{D} \emptyset$ Collaboration, "Evidence for an anomalous like-sign dimuon charge asymmetry," Phys. Rev. Lett. 105 (2010) pp. 081801, hep-ex arxiv:1005.2757.

[10] U. Nierste, "CP asymmetry in flavour-specific $B$ decays," hep-ph 0406300 v2 (2006).

[11] R. W. Lambert, "LHCb Hybrid Photon Detectors and sensitivity to flavour specific asymmetry in neutral B-meson mixing," CERN-THESIS-2009-001 (2008). 